\section{Emotion regulation involves both model-based and model-free processes}

\author{
Amit Etkin, Christian Büchel and James J. Gross
}

In response to our Opinion article (The neural bases of emotion regulation. Nat. Rev. Neurosci. 16, 693-700 (2015)) $)^{1}$, Raio et al. (Classifying emotion regulation strategies. Nat. Rev. Neurosci. http://dx.doi.org/10.1038/

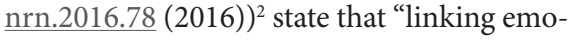
tion regulation to computational decision making is valuable", endorsing our position, and urge that "emotion regulation strategies should be classified on the basis of the degree to which they involve MB [model-based] elements (for example, prospection) and MF [model-free] elements (for example, linking action and reinforcement)." We strongly agree that emotion regulation may be best conceptualized as a mix of MF and MB processes. Indeed, we made this point in our paper (for example, see figure 3 of the paper).

One reason this point bears emphasizing is that although the task examples we used were meant to illustrate paradigms that more heavily involved MF or MB emotion regulation, none of them was developed to explicitly delineate their MF and MB components. Moreover, although it is unlikely that any task purely requires MF or MB control, comparing tasks that rely more heavily on MF or MB control may nonetheless be instructive for reframing emotion regulation in terms of value-based decision making (and associated computational models). For the field of emotion regulation to successfully adopt a computational framework, a new generation of paradigms will therefore need to be designed, which better operationalize and can experimentally manipulate MF and $\mathrm{MB}$ components.
Several related points are worth mentioning in this context. First, individuals are continually building models of their environment, suggesting that even processes that begin as entirely MF may evolve into primarily MB ones ${ }^{3}$. The balance and - as Raio et al. emphasize - the hierarchical relationship between $\mathrm{MF}$ and $\mathrm{MB}$ regulation are therefore dynamic and responsive to changes in the environment or individual. One important direction for work in this area is to delineate the temporal dynamics of the interplay between MF and MB processes.

Second, individuals appear to differ in their tendency to use MF or MB approaches, in both value-based decision making and emotion regulation. For example, individual differences in working memory influence the balance between $\mathrm{MF}$ and $\mathrm{MB}$ control ${ }^{4-6}$. It also appears that higher levels of presynaptic ventral striatal dopamine are associated with a bias towards MB learning ${ }^{7}$. In clinical contexts, patients displaying compulsive behaviour appear to be biased towards MF learning ${ }^{8}$. In future work, it will be important to more precisely characterize these individual differences in the emotion regulation domain both in normal and clinical-range variation.

Third, the repertoire of potential emotion regulation strategies available to the individual is diverse. Thus, MF and MB processes, their admixture and their hierarchical relationships are likely to vary across different strategies. As such, it is important to carefully operationalize the MF and MB elements of any emotion regulation strategy, and to capture these in experimental paradigms designed to facilitate computational modelling. Clearly, the mechanistic insights potentially afforded by adopting a value-based decision-making and computational modelling framework are substantial. We are therefore excited to see the field evolve in this direction, promising a new understanding of emotion regulation processes.

Amit Etkin is at the Department of Psychiatry and Behavioral Sciences, Stanford University, 401 Quarry Road, Stanford, California 94304, USA; and at the Veterans Affairs Palo Alto Healthcare System, and the Sierra Pacific Mental IIIness, Research, Education, and Clinical Center (MIRECC), 3801 Miranda Avenue, Palo Alto, California 94304, USA.

Christian Büchel is at the Department of Systems Neuroscience, University Medical Center HamburgEppendorf, 20246 Hamburg, Germany. Martinistr 52, 20246 Hamburg, Germany.

James J. Gross is at the Department of Psychology, Stanford University, 450 Serra Mall, Stanford, California 94305, USA.

Correspondence to A.E. amitetkin@stanford.edu doi: $10.1038 /$ nrn.2016.79 Published online 9 Jun 2016

1. Etkin, A., Buchel, C. \& Gross, J. J. The neural bases of emotion regulation. Nat. Rev. Neurosci. 16, 693-700 (2015).

2. Raio, C. M., Goldfarb, E., Lempert, K. \& SokolHessner, P. Classifying emotion regulation strategies. Nat. Rev. Neurosci. http://dx.doi.org/10.1038/ nrn.2016.78 (2016).

3. Dayan, P. \& Berridge, K. C. Model-based and modelfree Pavlovian reward learning: revaluation, revision, and revelation. Cogn. Affect. Behav. Neurosci. 14, 473-492 (2014).

4. Otto, A. R., Raio, C. M., Chiang, A., Phelps, E. A. ¿ Daw, N. D. Working-memory capacity protects model-based learning from stress. Proc. Natl Acad. Sci. USA 110, 20941-20946 (2013).

5. Schad, D. J. et al. Processing speed enhances modelbased over model-free reinforcement learning in the presence of high working memory functioning. Front. Psychol. 5, 1450 (2014).

6. Smittenaar, P., FitzGerald, T. H., Romei, V., Wright, N. D. ¿ Dolan, R. J. Disruption of dorsolateral prefrontal cortex decreases model-based in favor of model-free control in humans. Neuron 80, 914-919 (2013).

7. Deserno, L. et al. Ventral striatal dopamine reflects behavioral and neural signatures of model-based control during sequential decision making. Proc. Natl Acad. Sci. USA 112, 1595-1600 (2015).

8. Voon, V. et al. Disorders of compulsivity: a common bias towards learning habits. Mol. Psychiatry 20, 345-352 (2015).

Competing interests statement

The authors declare no competing interests. 